\title{
EVALUATION OF ACID DIFFUSION IN CHEMICALLY AMPLIFIED RESIST USING INTERDIGITATED ARRAY ELECTRODE.
}

\author{
Jiro NAKAMURA, Hiroshi BAN, and Akinobu TANAKA
}

NTT LSI Laboratories, 3-1, Morinosato Wakamiya, Atsugi-shi, Kanagawa 243-01

\begin{abstract}
We report on the evaluation of the catalytic acid behavior in chemically amplified resists, which was carried out using an interdigitated array electrode (IDA). When direct current is applied to resist films during the post-exposure baking process, the resist sensitivity decreases near the edge of the cathode on the IDA. This phenomenon is basically common to the several types of chemically amplified resists used in this work. It is considered that catalytic protons generated during exposure reduce, becoming electrically neutral on the cathode surface and losing their catalytic ability for chemical amplification reactions. Reduction causes a concentration gradient and the diffusional transport of protons. There is a semicircular cavity in a cross-sectional-developed pattern of chemically amplified negative type resists. From the size of the cavity, the acid diffusion coefficient in SAL601 (Shipley Co.) resist can be roughly estimated to be $70 \mathrm{~nm}^{2} / \mathrm{s}$ at $100^{\circ} \mathrm{C}$.
\end{abstract}

\section{Introduction}

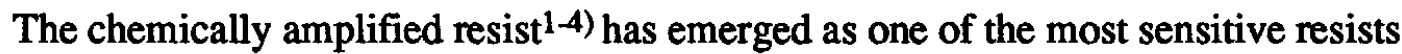
for deep-UV, electron beam and X-ray lithographies. Acids generated during exposure diffuse in resist film while acting as a catalyst for chemical amplification reactions. The diffusion of catalytic acid ${ }^{5-10}$ ) during post-exposure baking (PEB) has a strong dependence on the lithographic characteristics of chemically amplified resists. A trade-off between sensitivity and resolution attributed to acid diffusion in the chemically amplified resist has been reported ${ }^{10}$ ). With larger acid diffusion length, the resolution worsens and the 
sensitivity improves conversely. As a pattern rule becomes smaller, it becomes more important to quantitatively understand the range of acid diffusion in the resist so as to optimize process conditions and improve resist materials. For these purposes, simple methods for evaluating the acid movement in chemically amplified resists are required.

This paper describes the patterning of a chemically amplified resist on an interdigitated array electrode (IDA) ${ }^{11-14)}$. The IDA has been attracting much attention in various fields. The small electrodes of an interdigitated array enables the electrochemical measurement over a small region, and moreover it decreases the voltage drop even in low conductivity materials, enabling an evaluation of the movement of electroactive species in those materials. In this study, an IDA was used to evaluate the diffusion of catalytic protons in a chemically amplified resist. The patterning of chemically amplified resists was carried out on the IDA, applying direct current to the resist films during the PEB process. This paper also discusses the catalytic proton movement in the resist film on the basis of the developed pattern profile.

\section{Experimental}

The IDA used in this study is illustrated in Fig. 1. It was fabricated on Si substrate using photolithography. The anode and cathode material was $\mathrm{Pt}$. The width of each electrode and the gap between them was $2 \mu \mathrm{m}$. Several types of chemically amplified resists, including negative and positive resist, were used to evaluate the patterning characteristics on the IDA. The resist film was spincoated on the IDA and prebaked on a hot plate at $100^{\circ} \mathrm{C}$ for $120 \mathrm{~s}$. The resist film on the IDA was irradiated with an electron beam (EB) and was then post-exposure baked in an oven at $70-100^{\circ} \mathrm{C}$ for $30 \mathrm{~min}$ while the direct current bias of 3-30 V was applied. The resist film was developed with an aqueous tetramethyl-ammonium hydroxide solution. Moreover, the cross-sectional-development was used to clarify the change in film solubility in detail. After all areas of resist film on IDA were irradiated, the resist film on Si was cut orthogonal to the electrodes to obtain a cross section, and then it was developed. Using this method, the differences in dissolution rate can be evaluated more accurately than they can in conventional development because of lateral development of the resist film. Then a cross section of the resist pattern profile was observed by scanning electron microscopy (SEM).

\section{Results and Discussion}

A line pattern was delineated across the electrode while direct current bias of $10 \mathrm{~V}$ was applied to the resist film during the PEB process. The film thickness was $0.3 \mu \mathrm{m}$. The developed line patterns of SAL601 (Shipley Co.) are shown in Fig. 2. The width of resist pattern near the cathode was narrower than that near the anode. An example of a chemically amplified positive resist EXP4) pattern is shown in Fig. 3. EXP is composed of novolak 


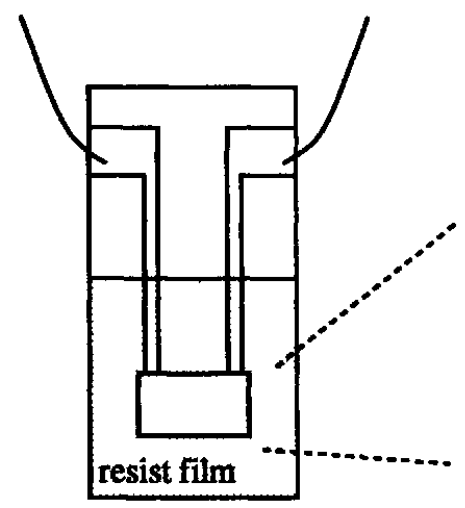

(a)



(b)

Fig. 1 (a) Illustration of the IDA electrode. (b) Detailed view of anodes and cathodes. The width of each electrode and the gap between them is $2 \mu \mathrm{m}$.

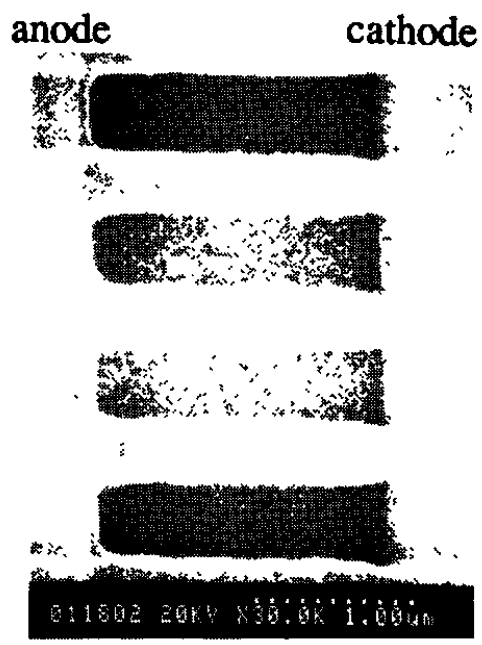

(a)

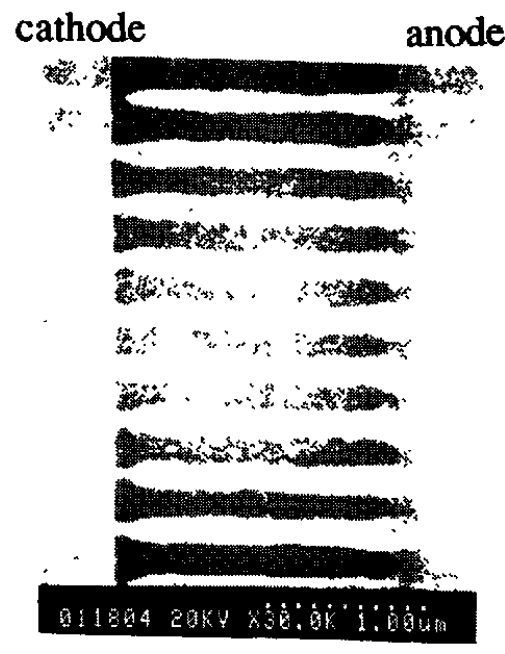

(b)

Fig. 2 SEM photograph of line and space pattern of SAL on the IDA. (a) $0.5-\mu \mathrm{m}$ line and space pattern. (b) $0.2-\mu \mathrm{m}$ line and space pattern. The exposure dose was 5 $\mu \mathrm{C} / \mathrm{cm}^{2}$ and the applied voltage was $10 \mathrm{~V}$.

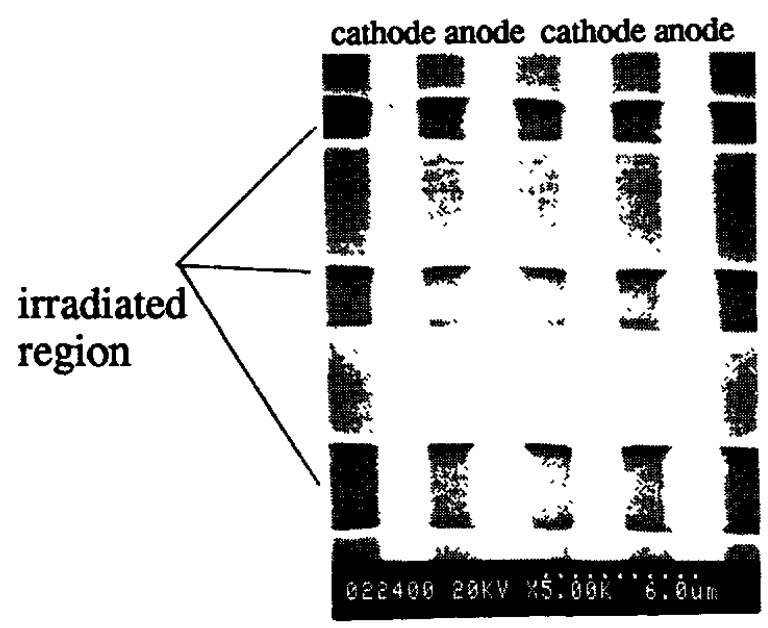

Fig. 3 SEM photograph of EXP pattern on the IDA. The exposure dose was $6 \mu \mathrm{C} / \mathrm{cm}^{2}$ and the applied voltage was $10 \mathrm{~V}$. 
resin, 2,2-bis(t-butoxy-carbonyloxyphenyl)-propane as a dissolution inhibitor and bis(p-tbutyphenyl)-iodonium trifluoromethanesulfonate as an acid generator. The resist film is not cleared on cathode, while the resist film on anode and between electrodes is cleared. This means that the resist sensitivity decreased near the cathode. This phenomenon is basically common in negative and positive resists. As the same material was used as the cathode and the anode, the effect of electron scattering from electrode substrate was equal in both electrodes. Therefore the decrease in sensitivity seems to be associated with catalytic acid behavior on the IDA. Examples of a cross-sectional developed resist pattern of SAL are shown in Fig. 4. The film thickness was $1.0 \mu \mathrm{m}$. A semicircular cavity with the radius of about $0.3 \mu \mathrm{m}$ can be seen in the resist near the edge of the cathode, while there is no visible change near the anode and between the anode and cathode. Moreover, the decrease in resist sensitivity in the edge of the cathodes was more remarkable than that in the center of the cathodes.

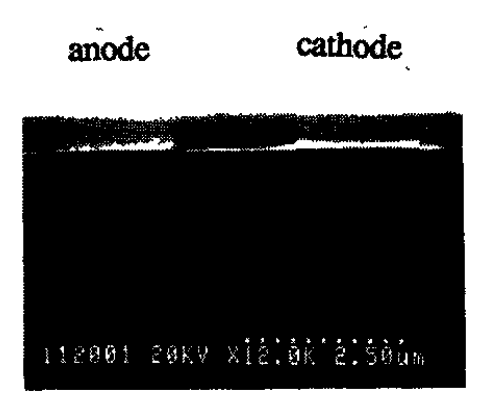

(a)

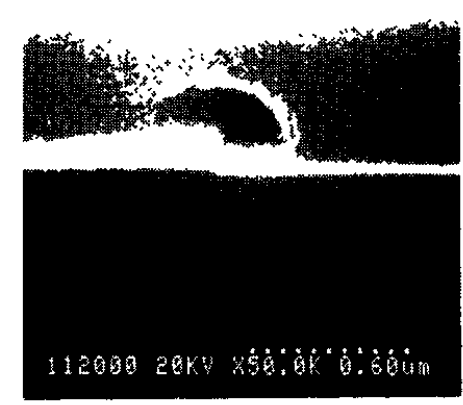

(b)

Fig. 4 (a) SEM photograph of crosssectional-developed pattern on the IDA. (b) A magnified view of cathode edge. All areas of resist film in IDA were irradiated. The exposure dose was $3 \mu \mathrm{C} / \mathrm{cm}^{2}$ and the applied voltage was $10 \mathrm{~V}$.

To clarify the effect of acid generator on the cavity, we prepared chemically amplified negative resists composed of novolak resin, alkai-soluble silicone polymer (ASSP) $^{15)}$, and acid generators. The acid catalyzed condensation of ASSP give a negative resist performance. Novolak resin was used to strengthen the resist pattern. The resist was prepared by using $1 \mathrm{~g}$ of novolak, $0.33 \mathrm{~g}$ of ASSP and $0.03 \mathrm{~g}$ of acid generator in 2ethoxyethyl-acetate. Triphenyl-sulfonium-hexafluoro-arsenate, triphenyl-sulfonium-fluorophosphate and triphenyl-sulfonium-triflate were used as acid generators. All of the acids used in this study caused the cavity on the cathode.

In a chemically amplified resist system, protons generated by exposure diffuse in resist film and act as a catalyst for chemical amplification reactions during the PEB process. The SAL resist system utilizes the change in the dissolution rate of resist film caused by the 
acid-catalyzed crosslinking reactions. According to an investigation ${ }^{16)}$ of the crosslinking reaction of the hydroxy-functional polymer with alkylated melamines, the rate equation can be represented by

$$
d[R-O H] / d t=-k[R-O H]\left[H^{+}\right]
$$

where $[R-O H]$ is the normalized concentration of the crosslinkable sites, $\left[H^{+}\right]$is the normalized concentration of catalytic proton, $k$ is the apparent reaction rate constant and $t$ is time. When the acid concentration changes due to acid diffusion, it should be regarded as a time-dependent factor. $[\mathrm{R}-\mathrm{OH}]$ can be, therefore, represented by

$$
\begin{aligned}
& {[R-O H]=\exp (-k M),} \\
& M=\int_{0}^{t_{P}[B}\left[H^{+}\right] d t
\end{aligned}
$$

where $t_{P E B}$ is the PEB time and $M$ represents the integral of acid concentration from 0 to the PEB time. With the same PEB and development conditions, the value of $M$ determines the density of the crosslinking reaction or, in other words, the dissolution rate of resist film for the developer. Consequently, the semicircular cavity in the edge of the cathode seems to be caused by a decrease in the density of the crosslinking reaction. This means the concentration of catalytic protons near the cathode decreases. Although here we take the SAL resist system by way of example to explain the rate equation of the chemical amplification reaction, the $M$ value basically determines the amount of chemical amplification reaction in other types of chemically amplified resists.

On the other hand, the modes of proton transfer in resist film are migration and diffusion ${ }^{17}$. Here, migration and diffusion mean the movement induced by an electric field and the movement caused by the influence of the concentration gradient, respectively. The proton transfer $J(x)$ to the cathode for one-dimensional transfer is expressed by

$$
J(x)=-D d C(x) / d x-(z F / R T) D C(x) d f(x) / d x
$$

where $D$ is the diffusion coefficient, $C(x)$ is the concentration at distance $x$ from the electrode, $z$ is the charge, $F$ is the faraday, $R$ is the gas constant, $T$ is absolute temperature and $d f(x) / d x$ is the potential gradient. Although we have not identified the electrochemical reaction on the cathode, the catalytic proton is assumed to be reduced to a hydrogen molecule on the cathode surface (Fig. 5). This reaction leads to a decrease in the catalytic proton concentration in the vicinity of the cathode. The concentration gradients of the protons arise and the diffusional transport of protons also occurs. As the potential distribution between electrodes and the interface between the resist film and electrode is 
unknown, it is difficult to distinguish between the migration and the diffusion. To clarify the effect of migration, the same experiment was carried out using an IDA whose anodes and cathodes were covered with a SiN membrane. Proton reduction is considered not to occur at the surface of $\mathrm{SiN}$. If a large migration occurs between the anodes and cathodes, catalytic protons move from the anodes to the cathodes. This will result in decreased proton concentration near the anodes and subsequently the generation of the cavity near the anode in a cross-sectional-developed pattern. There was, however, no cavity in the resist pattern on either the anode or cathode covered with SiN membrane. This phenomenon suggests that the proton flux to cathodes is mainly due to diffusion caused by concentration gradients. As proton reduction proceeds, the diffusion layer becomes longer. From the shape of the cavity in the resist, the configuration of the diffusion layer was concentric near the cathode edge. This suggests that more proton reduction occurred in the edge than in the center of the cathode. On the other hand, the counter anion is assumed to be oxidized on the anode surface. No cavity was seen in the cross-sectionaldeveloped pattern near the anode. The effect of the counter anion on the crossliking reaction can be deduced to be small.

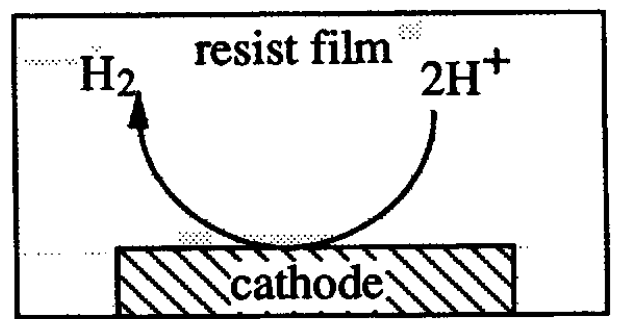

Fig. 5

Mechanism of the electrochemical reaction on the cathode surface.

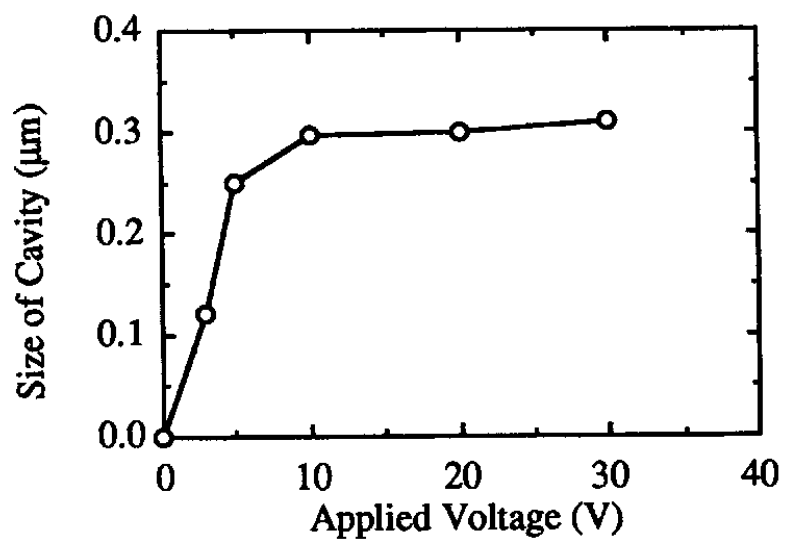

Fig. 6 Dependence of cavity size on applied valtage.

The dependence of cavity size on the applied voltage is shown in Fig. 6. The size becomes larger with increased voltage and saturated to be about $0.3 \mu \mathrm{m}$ at over $10 \mathrm{~V}$. In electrochemistry, the Nernst equation, 


$$
E=E_{0^{\prime}}+(R T / n F) \ln ((O] /[R]) \quad(5)
$$

is relied to provide a linkage between electrode potential $\mathrm{E}$ and the concentrations of electroactive species. Here $E_{0^{\prime}}$ is the formal potential of an electrode and $O$ and $R$ are oxidized species and reduced species, respectively. As the applied voltage is larger, the concentration of protons at the cathode surface is smaller and reaches zero. In this case, proton reduction is determined by the rate of proton transfer to the cathode. If the voltage is stepped to the mass-transfer region, the concentration of protons becomes nearly zero at the cathode surface. The rate of proton transfer is proportional to the concentration gradient at electrode surface. Therefore, the cavity size is independent of applied voltage over $10 \mathrm{~V}$.

Spreading of the diffusion layer caused by proton reduction at the cathode is proportional to the square root of the diffusion coefficient of protons in the resist film and PEB time. Assuming that the cavity radius is equal to the length of the diffusion layer $(\sqrt{2 \mathrm{Dt}}$ ), the diffusion coefficient of protons can roughly be estimated to be about $70 \mathrm{~nm}^{2} / \mathrm{s}$, which corresponds to results in previous papers. ${ }^{18,19)}$ With this diffusion coefficient, acid diffusion hardly influences the profile in quartermicron patterns at the conventional PEB time of $120 \mathrm{~s}^{10)}$, however, the profile in smaller patterns degrades due to acid diffusion.

\section{Conclusion}

Patterns of the chemically amplified resists were fabricated on IDAs with voltage being applied during the PEB process. The resist sensitivity on the cathode was smaller than that on the anode. This phenomenon is observed in the various chemically amplified resists used in this work. Cross-sectional development was used to evaluate the sensitivity change in the cathode in detail. There was a semicircular cavity with a radius of about 0.3 $\mu \mathrm{m}$ in the cross-sectional-developed pattern near the cathode edge. The decrease in resist sensitivity near the cathode was considered to be due to proton reduction on the cathode surface. Catalytic protons reduced, becoming electrically neutral and losing the catalytic ability for chemical amplification reactions. The reduction created a concentration gradient of protons that produces a subsequent flux of protons to the electrode surface and the diffusion layer in the concentric configuration about the cathode edge. The diffusion coefficient of protons in SAL resist was roughly estimated to be about $70 \mathrm{~nm}^{2} / \mathrm{s}$. As the size of cavity depends on the diffusion layer, this IDA experiment will be useful tool for evaluating acid diffusion in a chemically amplified negative resist.

\section{Acknowledgement}


The authors wish to thank Tetsushi Sakai and Tadahito Matsuda for their advice and encouragement. They also thank Masao Morita, Hisao Tabei and Osamu Niwa for their helpful discussion on the IDA experiment.

\section{References}

1) H. Ito and C. G. Willson: Polym. Eng. Sci. 23 (1983) 1012.

2) H. Liu, M. P. deGrandpre and W. E. Feely: J. Vac. Sci. \& Technol. B7 (1988) 379.

3) J. Lingnau, R. Dammel and J. Theis: Polym. Eng. Sci. 29 (1989) 874.

4) H. Ban, J. Nakamura, K. Deguchi, and A. Tanaka: J. Vac. Sci. \& Technol. B9 (1991) 3387.

5) D. R. McKean, U. Schaedeli and S. A. MacDonald: ACS Symp. Ser. 412 (1989) 27.

6) L. Schlegel, T. Ueno, N. Hayashi and T. Iwayanagi: J. Vac. Sci. \& Technol. B9 (1991) 3387.

7) J. Nakamura, H. Ban, K. Deguchi and A. Tanaka: Jpn. J. Appl. Phys. 30 (1991) 2619.

8) T. Fedynyshyn, M. Cronin and C. Szmanda: J. Vac. Sci. \& Technol. B9 (1991) 3380.

9) P. Trefonas and M. T. Allen: Proc. SPIE 1672 (1992) 74.

10) J. Nakamura, H. Ban and A. Tanaka: Jpn. J. Appl. Phys. 31 (1992) 4294.

11) D. G. Sanderson and L. B. Anderson: Anal. Chem., 57 (1985) 2388.

12) C. E. Chidsey, B. J. Feldman, C. Lundgren and R. W. Murray: Anal. Chem., 58 (1986) 601.

13) O. Niwa, M Morita, and H. Tabei: J. Electroanal. Chem., 267 (1989) 291.

14) M. Takahashi, M. Morita, O. Niwa, and H. Tabei: J. Electroanal. Chem., 335 (1982) 253.

15) A. Tanaka, H. Ban, J. Nakamura, and Y. Kawai: Jpn. J. Appl. Phys. 31 (1992) 2277.

16) D. R. Bauer and G. F. Budde: J. Appl. Polym. Sci. 28 (1983)253.

17) A. J. Bard and L. R. Faulkner " Electrochemical Methods" (John Wiley \& Sons, New York), Chap. 1.

18) J. Nakamura, H. Ban and A. Tanaka: J. Photopolym. Sci. \& Techol. 4 (1991) 83.

19) H. Yoshino and H. Matsumoto: Jpn. J. Appl. Phys. 31 (1992) 4283. 